\title{
BARHL2 Differentially Regulates the Development of Retinal Amacrine and Ganglion Neurons
}

\author{
Qian Ding, ${ }^{1,2}$ Hui Chen, ${ }^{5}$ Xiaoling Xie, ${ }^{1}$ Richard T. Libby, ${ }^{1,2}$ Ning Tian, ${ }^{5}$ and Lin Gan ${ }^{1,3,4}$ \\ ${ }^{1}$ University of Rochester Eye Institute, ${ }^{2}$ Department of Biomedical Genetics, ${ }^{3}$ Center for Neural Development and Disease, and ${ }^{4}$ Department of \\ Neurobiology and Anatomy, University of Rochester, Rochester, New York 14642, and ${ }^{5}$ Department of Ophthalmology and Visual Science, Yale University \\ School of Medicine, New Haven, Connecticut 06520
}

Through transcriptional regulations, the BarH family of homeodomain proteins play essential roles in cell fate specification, cell differentiation, migration, and survival. Barhl2, a member of the Barh gene family, is expressed in retinal ganglion cells (RGCs), amacrine cells (ACs), and horizontal cells. Here, to investigate the role of Barhl2 in retinal development, Barhl2-deficient mice were generated. Analysis of AC subtypes in Barhl2-deficient retinas suggests that Barhl2 plays a critical role in AC subtype determination. A significant reduction of glycinergic and GABAergic ACs with a substantial increase in the number of cholinergic ACs was observed in Barhl2-null retinas. Barhl2 is also critical for the development of a normal complement of RGCs. Barhl2 deficiency resulted in a 35\% increase in RGCs undergoing apoptosis during development. Genetic analysis revealed that Barhl2 functions downstream of the Atoh7-Pou4f3 regulatory pathway and regulates the maturation and/or survival of RGCs. Thus, BARHL2 appears to have numerous roles in retinal development, including regulating neuronal subtype specification, differentiation, and survival.

\section{Introduction}

In retina, visual stimuli are received by photoreceptor cells and transferred via bipolar cells to retinal ganglion cells (RGCs). The two types of interneurons, amacrine and horizontal cells, function to integrate and modulate visual signal processing within retina. Based on their unique morphological, physiological, neurotransmitter, and connectivity properties, retinal neurons are further divided into subtypes. For instance, amacrine cells (ACs), which reside in the amacrine cell layer (ACL), an inner layer of the inner nuclear layer (INL), and the ganglion cell layer (GCL), synapse with bipolar cells and RGCs. They constitute the most diverse cell type within the retina with more than 26 different subtypes based on their sublaminar localization (e.g., displaced ACs), morphology (e.g., starburst), and neurotransmitter types (e.g., GABAergic, dopaminergic, and cholinergic) (Masland, 2001a,b). Similarly, RGCs, the output neurons of the retina, are divided into more than 10 different subtypes based on their morphology, physiological properties, and stratification patterns (Kong et al., 2005). Although we have made significant progresses understanding the molecules important in generating the broad classes of retinal cell types, currently, the molecules defining retinal subtypes are poorly understood.

Previous studies have demonstrated that many transcription factors (TFs) play important roles in retinogenesis. During the

\footnotetext{
Received 0ct. 28, 2008; revised Jan. 31, 2009; accepted Feb. 12, 2009.

This work was supported by National Institutes of Health Grants EY013426 (to L.G.) and EY012345 (to N.T.), and the Research to Prevent Blindness challenge grant to the Department of Ophthalmology at the University of Rochester. We thank Dr. Alexandra Joyner for the W4 mouse ES cells and Dr. Amy Kiernan and the members of the Gan laboratory for many helpful discussions and technical assistance.

Correspondence should be addressed to Lin Gan at the above address. E-mail: lin_gan@urmc.rochester.edu. D01:10.1523/JNEUROSCI.5237-08.2009

Copyright $\odot 2009$ Society for Neuroscience $\quad 0270-6474 / 09 / 293992-12 \$ 15.00 / 0$
}

development of RGCs, ATOH7 (MATH5), a basic helix-loophelix (bHLH) TF, endows retinal precursors with the competency to form RGCs (Yang et al., 2003). In Atoh7-null retinas, $>95 \%$ RGCs fail to form (Brown et al., 2001; Wang et al., 2001). POU4F2, a POU-homeodomain (HD) TF, and ISL1, an LIM-HD $\mathrm{TF}$, are synergistically required for RGC differentiation and survival (Gan et al., 1999; Pan et al., 2008). Nevertheless, the loss of RGCs in mice lacking Pou $4 f 2$ or Atoh7 is not selective to any particular subtype (Lin et al., 2004), suggesting that they are general factors in RGC development. Among the key TFs in amacrine development, NEUROD4 and NEUROD1, both bHLH TFs, are redundantly required for amacrine genesis (Marquardt et al., 2001; Inoue et al., 2002), whereas retina-specific deletion of Pax6 HD TF results in the formation of only ACs (Marquardt et al., 2001). Mice lacking the winged helix/forkhead TF FOXN4 have fewer ACs and no horizontal cells (Li et al., 2004). However, the above TFs regulate AC generation in a nonsubtype-specific manner. Recently, studies show that BHLHB5, a bHLH TF, and ISL1 are expressed in GABAergic and cholinergic ACs, respectively, and are necessary for their differentiation (Feng et al., 2006; Elshatory et al., 2007), suggesting that unique TFs determine amacrine subtype development.

Bar-class HD (BarH) proteins are evolutionarily conserved and play essential roles in organogenesis. In Drosophila, BarH1 and $\mathrm{BarH} 2$ are expressed in $\mathrm{R} 1 / 6$ photoreceptor cells and are required for their differentiations (Higashijima et al., 1992). The Xenopus BarH orthologue, Xbh1, promotes RGC differentiation (Patterson et al., 2000; Poggi et al., 2004). In mouse retina, BARHL2 expression is found in RGCs, ACs, and horizontal cells. Forced expression of BARHL2 promotes the differentiation of glycinergic ACs at the expense of bipolar and Müller cells (Mo et al., 2004). Thus, BARHL2 appears to be an important molecule in 
mammalian retinal development. To understand the role of BARHL2 in retinal development, we generated a nonfunctional allele of Barhl2 in mice. In Barhl2-null retinas, there was a selective loss of RGCs at late embryonic stages. Moreover, there was an overall loss of ACs that included a significant loss of both glycinergic and GABAergic in Barhl2-null retinas. Interestingly, the number of cholinergic ACs was greatly increased. These data demonstrate that BARHL2 is an important factor for the maintenance of RGCs and ACs, as well as being critical for subtype specification of retinal neurons.

\section{Materials and Methods}

Generation and genotyping of Barhl2-lacZ and Barhl2-Cre mice. To generate Barhl2-null mice, we used Barhl2-coding sequences as a probe to isolate genomic sequences from mouse 129S6 BAC genomic library (CHORI). The Barhl2-lacZ reporter and Barhl2-Cre knock-in construct were generated by inserting $3.5 \mathrm{~kb}$ Barhl2 $5^{\prime}$ flanking sequences ending at the 20th nucleotide upstream of the initiation codon ATG and $3.5 \mathrm{~kb} 3^{\prime}$ sequence starting from the 68th nucleotide after ATG into $5^{\prime}$ and $3^{\prime}$ multiple cloning sites of pKI-lacZ and pKII-Cre vectors (L. Gan, unpublished observation) at the HindIII and EcoRI sites, respectively. The insertion placed lac $Z$ or Cre under the control of endogenous Barhl2 regulatory sequences. To generate Barhl2-null mice, AscI-linearized Barhl2lacZ- and Barhl2-Cre-targeting constructs were electroporated into W4 mouse embryonic stem (ES) cells (Auerbach et al., 2000) and positivetargeted ES cells were obtained by drug selection with G418 and FIAU. The targeted ES cells were confirmed by Southern blotting genotyping and were injected into $\mathrm{C} 57 \mathrm{BL} / 6 \mathrm{~J}$ blastocysts to generate mouse chimeras. Barhl2-lacZ and Barhl2-Cre mice were generated in 129 S6 and C57BL/6J mixed background as described previously (Gan et al., 1996, 1999). PCR was performed to genotype mice from heterozygous mating. PCR primers used to identify the wild-type (WT) Barhl2 allele are 5'ATGACAGCACTGGAAGGGGCC- $3^{\prime}$ and $5^{\prime}$-ACGCTGGTGCTGTAGGGTGCACA- $3^{\prime}$; the lac $Z$ allele $5^{\prime}$-AGGGCCGCAAAACTATCC- $3^{\prime}$ and $5^{\prime}$-ACTTCGGCACCTTACGCTTCTTCT-3'; and the Cre allele 5' GCAGTGTCAACGCTTTTTAGTGTC- ${ }^{\prime}$ and $5^{\prime}$-CCATGAGTGAACGAACCTGGTCG-3'. The Z/EG conditional green fluorescent protein (GFP) reporter mice were purchased from The Jackson Laboratory (Stock number: 003920), and genotyping was performed according to protocols provided by The Jackson Laboratory. Embryos were identified as E0.5 at noon on the day at which vaginal plugs were first observed. All animal procedures in this study were approved by University Committee of Animal Resources at University of Rochester.

Immunohistochemistry, in situ hybridization, and X-Gal staining. Staged mouse embryos and enucleated eyes of postnatal mice were fixed with $4 \%$ paraformaldehyde in $\mathrm{PBS}$ for $1-2 \mathrm{~h}$ at $4^{\circ} \mathrm{C}$. After the fixation, samples were cryopreserved with $20 \%$ sucrose, embedded in OCT compound, and cryosectioned at a thickness of $14 \mu \mathrm{m}$. Immunostaining, in situ hybridization, and X-Gal staining were performed as described previously (Pan et al., 2005). For whole-mount immunostaining, animals were anesthetized with halothane and perfused with $4 \%$ paraformaldehyde in PBS. Eyecups were isolated and blocked in $10 \%$ horse serum with $0.5 \%$ Triton X-100 for $4 \mathrm{~h}$ and incubated with primary antibody for $2-3$ $\mathrm{d}$ at $4^{\circ} \mathrm{C}$. Then, eyecups were washed in PBS and incubated with fluorescently labeled secondary antibody for $1 \mathrm{~d}$ at $4^{\circ} \mathrm{C}$. After three rinses of 15 min each, retinas were dissected out and mounted in PBS on slides. Retinal images were captured with a Zeiss LSM 510 META confocal microscope. To quantify the numbers of immunolabeled cells, five or more retinas for each genotype were analyzed with Image J software (National Institutes of Health).

The following antibodies and dilutions were used: rabbit anti- $\beta$ galactosidase (lacZ) (1:500; Millipore Bioscience Research Reagents), goat anti-POU4F2 (1:200; Santa Cruz), mouse anti-calbindin (1:2000; Sigma), mouse anti-POU4F1 (1:200; Millipore Bioscience Research Reagents), mouse anti-syntaxin (1:5000; Santa Cruz), mouse anti-PAX6 [1:200; Developmental Studies Hybridoma Bank(DSHB)], mouse antiIsl1/2 (1:200; DSHB), goat anti-ChAT (1:100; Millipore Bioscience Research Reagents), sheep anti-Chx10 (Exalpha), rabbit anti-PKC $\alpha$
(1:5000; Sigma), mouse anti-GAD65 (1:200; BD Bioscience), rabbit antiactivated caspase3 (1:200; BD PharMingen), goat anti-GLYT1 (1:5000; Millipore Bioscience Research Reagents), rabbit anti-opsin (1:200), chicken anti-GFP (1:500; Abcam). Alexa-conjugated secondary antibodies (Invitrogen) were used at a concentration of 1:500.

Multielectrode array recording. The procedures of retina preparation, action potential recording, and data analysis have been described previously in detail (Tian and Copenhagen, 2003). Briefly, mice were dark adapted for $30 \mathrm{~min}$ before being killed. Retinas were isolated under infrared illumination in oxygenated extracellular solution, which contained (in mM) $124 \mathrm{NaCl}, 2.5 \mathrm{KCl}, 2 \mathrm{CaCl}_{2}, 2 \mathrm{MgCl}_{2}, 1.25 \mathrm{NaH}_{2} \mathrm{PO}_{2}, 26$ $\mathrm{NaHCO}_{3}$, and glucose $22\left(\mathrm{pH} 7.35\right.$ with $95 \% \mathrm{O}_{2}$ and $5 \% \mathrm{CO}_{2}$ ). Isolated retina was mounted in the multielectrode array (MEA-60) chamber with the ganglion cell layer facing the recording electrodes and continuously perfused with oxygenated extracellular solution at $34^{\circ} \mathrm{C}$. For spontaneous retinal waves, data were collected for $30 \mathrm{~min}$. For light-evoked action potentials, a white rectangular moving bar $(600 \times 4000 \mu \mathrm{m})$ was used to stimulate the retinas, while moving on a black background and perpendicular to its long axis at $1000 \mathrm{~mm} / \mathrm{s}$ and in 12 directions at $30^{\circ}$ intervals with a pseudorandom sequence. The stimulations were repeated $30 \times$ with random sequences. Data were collected using a PC-based interface card and software (Multi Channel System). The signals were filtered between $100 \mathrm{~Hz}$ (low cutoff) and 200 or $250 \mathrm{kHz}$ (high cutoff). Off-line data analysis was performed on a PC computer using off-line sorter (Plexon).

MEA data analysis. For spontaneous retinal waves, the cell with highest firing rate was selected for each electrode for the calculation of correlation index. The correlation index for a given pair of neurons was calculated based on the following equation (Wong et al., 1993; Torborg et al., 2005):

$$
\text { Correlation index }=\frac{N_{\mathrm{AB}(-0.1 \mathrm{~s}, 0.1 \mathrm{~s})} \times T}{N_{\mathrm{A}(0, \mathrm{~T})} \times N_{\mathrm{B}(0, \mathrm{~T})} \times(0.2 \mathrm{~s})},
$$

where $N_{\mathrm{AB}}$ is the number of spike pairs from cells $\mathrm{A}$ and $\mathrm{B}$ for which cell B fires within $\pm 100 \mathrm{~ms}$ of cell $\mathrm{A}, T$ is the total recording time, $N_{\mathrm{A}(0, \mathrm{~T})}$ is the total number of spikes in cell $\mathrm{A}$, and $N_{\mathrm{B}(0, \mathrm{~T})}$ is the total number of spikes in cell $\mathrm{B}$. The frequencies of action potentials of all sorted cells were averaged to calculate the average firing rate. The interwave interval is the time between the two subsequent waves determined manually based on the spike trains.

For light-evoked directional selective responses, self-programmed software was used to calculate the peak spike frequency of leading and trailing edge (ON and $\mathrm{OFF}$ ) responses for each cell. The responses were averaged from 30 recording trials. The directional selectivity index (DSI) was calculated as follows:

$$
\text { DSI }=\frac{\text { Preferred peak frequency }- \text { Null peak frequency }}{\text { Preferred peak frequency }+ \text { Null peak frequency }} .
$$

\section{Results}

\section{Targeted deletion of Barhl2 and retinal expression of lacZ} knock-in reporter gene

To investigate the role of Barhl2 in retinal development, we generated a targeted deletion of Barhl2 by homologous recombination (supplemental Fig. S1 $A, B$, available at www.jneurosci.org as supplemental material). The Barhl2 ${ }^{\text {lac } Z}$ knock-in allele was created by replacing $88 \mathrm{bp}$ in exon 1 with the lac $Z$ reporter gene and SV40 polyadenylation sequences to prevent the transcription of the remaining Barhl2-coding sequences. In situ hybridization, using Barhl2 antisense probe, showed that the expression of Barhl2 was abolished in Barhl2 ${ }^{\text {lacZ/lacZ }}$ (Barhl2 null) retinas and anti-lacZ immunostaining revealed the expression of lacZ in the embryonic retinas (supplemental Fig. S1C, available at www. jneurosci.org as supplemental material). The heterozygous Barhl2 $2^{\text {lacZ/+ }}$ mice were indistinguishable from the WT and, therefore, included as controls. Offspring from the Barhl2 $2^{\text {lacZ/+ }}$ intercross were born in a standard Mendelian ratio. Out of the first 289 
mice weaned, $22.5 \%$ were WT, $52.9 \%$ were Barhl2 ${ }^{\text {lacZ/+ }}$, and $24.6 \%$ were Barhl2 $2^{\text {lacZ/lacZ }}$. Barhl2-nulls (Barhl2 ${ }^{\text {lacZ/lac } Z}$ ) mice were indistinguishable from littermate controls at birth. However, they did not gain weight at the same rate as controls and gradually displayed signs of impaired motor coordination. Bahrl2-null mice died before postnatal day 24 (P24).

Previous studies have shown that the earliest expression of Barhl2 in mouse retina is at E13.5 in the inner neuroblastic layer (NBL). In the adult retina, Barhl2 is expressed in amacrine, horizontal, and ganglion cells (Mo et al., 2004). To examine the retinal cell types that Barhl2-lacZ is expressed in and to determine whether the expression pattern of lac $Z$ reflects that of endogenous Barhl2, we doubleimmunolabeled retinas from Barhl2 $2^{\text {lacZ/+ }}$ mice with anti-lacZ antibody and specific cell type-specific markers. LacZ + cells were in three distinct locations in the retina, in the GCL and the inner and outer portions of the INL. In the GCL, LacZ+ cells colabeled with an RGC marker, POU4F1, in some RGCs (supplemental Fig. S1 $D$, available at www.jneurosci.org as supplemental material). All Barhl2expressing cells in the GCL and the inner portions of the INL colabeled with PAX6, a marker for all RGCs and ACs (supplemental Fig. S1E, available at www.jneurosci. org as supplemental material). Double staining with amacrine subtype-specific markers revealed that lac $Z$ was expressed in all cholinergic ACs (ISL1+), selective glycinergic amacrine (GLYT1+), and GABAergic (GAD65+) ACs (supplemental Fig. $\mathrm{S} 1 \mathrm{~F}-\mathrm{H}$, available at www. jneurosci.org as supplemental material). At the outer border of the INL, all horizontal cells (calbindin + ) expressed lacZ (supplemental Fig. S1 $I$, available at www. jneurosci.org as supplemental material). The absence of colabeling of lac $Z$ with CHX10, opsin, and GFAP excluded the possibility that Barhl2 is expressed in bipolar, photoreceptor, and glial cells, respectively (data not shown). These results are consistent with the expression pattern of Barhl2 reported previously (Mo et al., 2004) and indicated that the knock-in lacZ allele recapitulated endogenous Barhl2 expression.

\section{Retinal defects in Barhl2-null mice}

To investigate the role of Barhl2 in retinal development, we first assessed the retinal histology of Barhl2-null retinas at different developmental stages. Before E15.5, no alterations in retinal laminar organization and thickness were apparent in Barhl2 nulls (Fig. 1A). Starting at E16.5, Barhl2-null retinas had a noticeable reduction in GCL thickness, and by P7, the INL thickness was also reduced (Fig.

$G$
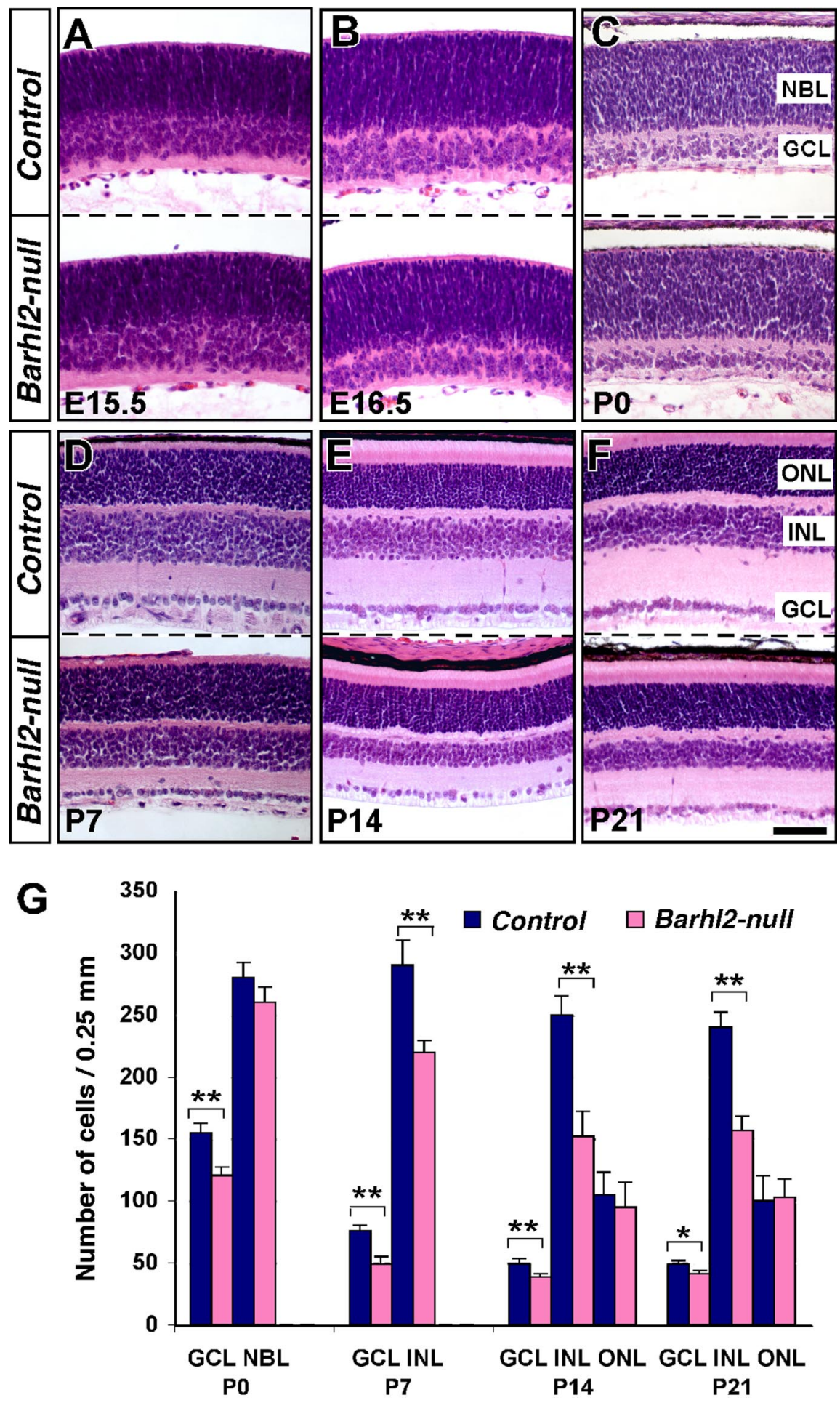

Figure 1. Developmental abnormality of Barh/2-null retinas. Retinas from control and Barh/2-null mice were analyzed by hematoxylin and eosin staining at different developmental stages. $\boldsymbol{A}$, Compared with the controls, no overt change in retinal thickness and laminar structures is observed in Barh/2-null retinas before E15.5. B-F, From E16.5, the GCL of Barh/2-null retina is thinner, and the number of cells in the GCL is significantly reduced. This hypoplasia is maintained till P21. From P7, the INL also exhibits a marked reduction in cell numbers; at P21, the cell number in the INL is reduced $35.6 \%$. The ONL thickness is normal. $\mathbf{G}$, Quantitation of cells in the GCL and INL per $250 \mu \mathrm{m}$ length of retinal section at PO -P21. Cell counting in NBL in Cand ONL in $E$ and $\boldsymbol{F}$ covers $62.5 \mu \mathrm{m}$ linear length. ${ }^{*}$ t test $p<0.01 ;{ }^{* *}$ t test $p<0.001$. Scale bar, $50 \mu \mathrm{m}$.

$1 B-D)$. Although the overall laminar structure of Barhl2-null retinas resembled those of the controls at the completion of retinogenesis (at P21), Barhl2-null retinas had $16.3 \pm 2 \%$ fewer cells in the GCL and $35.6 \pm 6 \%$ less cells in the INL (Fig. $1 E-G$ ). 

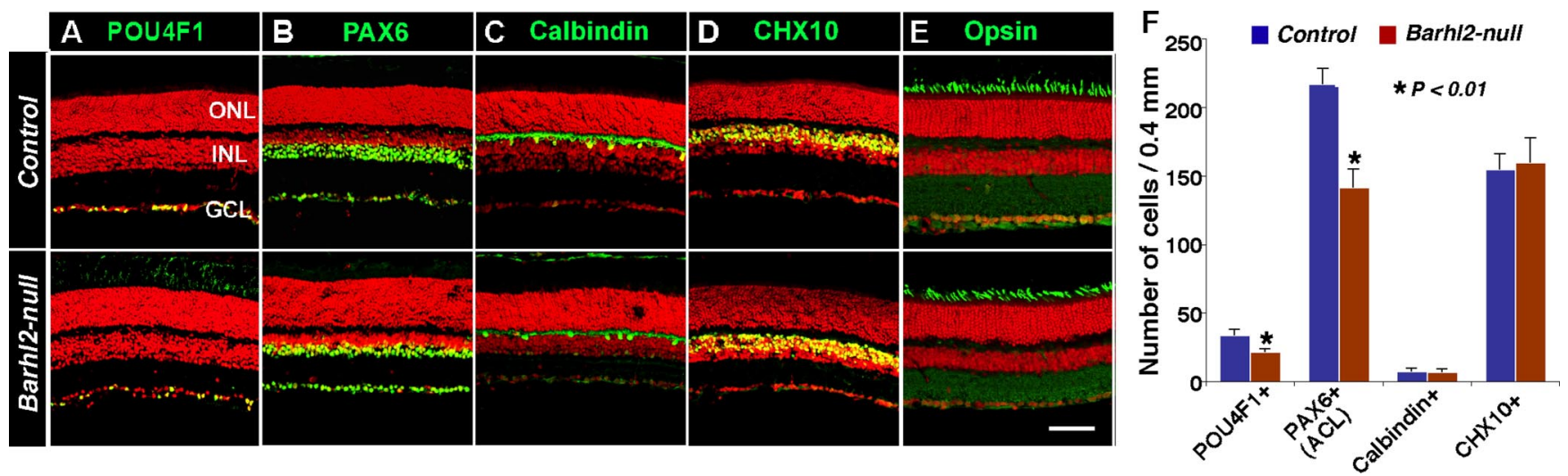

Figure 2. Effect of Barhl2-null mutation on RGCs and ACs in P21 retinas. Retinas were immunolabeled with indicated antibodies (green) and nuclear counterstained with propidium iodide (red). $A$, Loss of Barhl2 leads to a severe loss of RGCs immunoreactive for POU4F1 (average number of POU4F1+RGCs \pm SD: controls, $33 \pm 5, n=4 ; B$ arhl2 nulls, $22 \pm 3, n=4 ; p<0.01$, Student's $t$ test). $\boldsymbol{B}$, Loss of amacrine cells immunoreactive for PAX6 (average number of PAX6 + ACS \pm SD: controls, $217 \pm 11, n=4$; Barhl2 nulls, $142 \pm 13, n=4 ; p<0.01$, Student's $t$ test). $C-E$, No significant alteration in horizontal cells immunoreactive for calbindin ( $\boldsymbol{C}$, average number of calbindin + horizontal cells \pm standard SD: controls, $8 \pm 2, n=4 ; B$ arhl2 nulls, $7 \pm 2, n=4 ; p=0.63$, Student's $t$ test), bipolar cells immunoreactive for CHX10 ( $\boldsymbol{D}$, average number of CHX10+ bipolar cells \pm SD: controls, $155 \pm 11, n=4 ;$; Barhl2 nulls, $161 \pm 17, n=4 ; p=0.75$, Student's $t$ test), and photoreceptors immunoreactive for 0 psin $(\boldsymbol{E}) . \boldsymbol{F}$, Quantitation of immunoreactive cells per $250 \mu \mathrm{m}$ length of retinal section at P21. ${ }^{*} t$ test $p<0.01$. Scale bar, $100 \mu \mathrm{m}$.
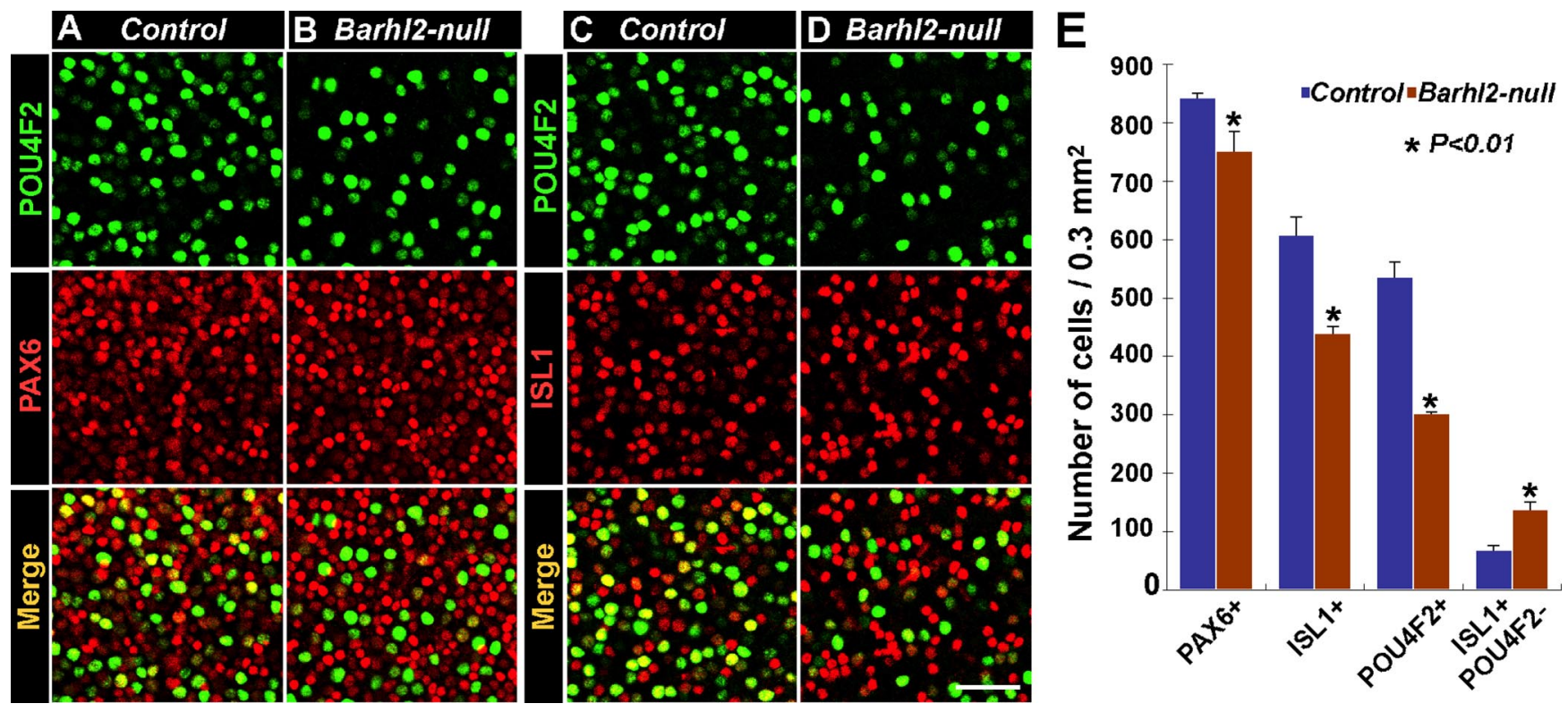

Figure 3. Analysis of RGCs and displaced ACs in the GCL of Barh/2-null retina. $A-D$, Retinas from control and Barh/2-null mice at P21 were coimmunolabeled with anti-PAX6 (red) and anti-P0U4F2 (green) $(\boldsymbol{A}, \boldsymbol{B})$ or with anti-ISL1 (red) and anti-P0U4F2 (green) $(\boldsymbol{C}, \boldsymbol{D})$. $\boldsymbol{E}$, Quantitation of immunolabeling results. In the GCL of Barh/2-null retina, the total number of ACs and RGCs labeled by anti-PAX6 and anti-ISL1 are reduced by 13.8 and 28.3\%, respectively. Specifically, the POU4F2-immunoreactive RGCs are decreased to 53\%, whereas the POU4F2-negative and ISL1-positive cells are increased more than twofold. Cell numbers were counted in the central regions of at least three independent samples from each genotype. ${ }^{*} t$ test $p<0.01$. Scale bar, $50 \mu \mathrm{m}$.

The expression of Barhl2 in amacrine, horizontal, and ganglion cells, and the marked loss of cells in the GCL and INL in Barhl2-null retinas, suggested that deletion of Barhl2 could impair the differentiation and or survival of neurons in the GCL and INL. To test this possibility, cell type-specific markers were used to analyze the development of retinal cell types and subtypes. Immunostaining for POU4F1, which labels $\sim 70 \%$ of RGCs (Xiang et al., 1993), revealed a 35\% decrease of these cells in the GCL (Fig. 2A). In the INL, anti-PAX6 labeling revealed a 39\% loss of ACs (Fig. 2 B). No significant changes in horizontal (calbindin +$)$, bipolar $(\mathrm{CHX10+})$, and photoreceptor $(\mathrm{Opsin}+)$ cells (Fig. 3C-F). In summary, the targeted deletion of Barhl2 selectively affects the development of RGCs and ACs but not horizontal cells that also express Barhl2.
Developmental loss of RGCs in Barhl2-null retinas

The loss of RGCs in Barhl2-null retinas prompted us to analyze further the changes in cell composition in the GCL. Wholemount immunostaining of the adult retinas revealed an $\sim 47 \%$ decrease of POU4F2 + RGCs (Fig. 3A-E) and a $13.8 \pm 4 \%$ decrease in PAX6+ cells (Fig. $3 A, B, E$ ) in Barhl2 nulls. Although ISL1 immunostaining for RGCs and cholinergic ACs revealed a $28.3 \pm 3.0 \%$ loss of all ISL1 + cells in the GCL, the POU4F2- and ISL1+ cells (presumably the displaced cholinergic ACs) increased by more than twofold (Fig. 3C-E), suggesting that the loss of RGCs in Barhl2 nulls is accompanied by an increase in the displaced ACs in the GCL, particularly the cholinergic amacrine subtypes.

To investigate whether the loss of RGCs results from a dys- 
regulation of neurogenesis or cell death, we next tested the generation of RGCs in the absence of Barhl2. POU4F2 expression marks the majority of RGCs during embryogenesis (Young, 1985; Gan et al., 1999). Immunostaining of POU4F2 revealed no change in the number of RGCs in Barhl2-null retinas before E15.5 (Fig. $4 A, B, M)$, suggesting that the genesis of RGCs was not affected in Barhl2 nulls. However, a 19.4\% decrease of POU4F2+ RGCs was observed starting at E16.5 in Barhl2-null retinas compared with controls (Fig. 4C,D,M). The number of POU4F2 + RGCs continued to decrease with age (Fig. $4 E-H, M$ ). Consistently, the reduction of RGCs was confirmed by a $33.7 \%$ reduction in the cross-section areas of the Barhl2-null optic nerves (Fig. 4I-L). To determine whether the loss of RGCs resulted from apoptosis in Barhl2-null retinas, we immunolabeled retinas with antiactivated CASP3, one of the main effectors in developmental cell death (Kuida et al., 1996). Barhl2-null retinas showed a gradual increase of apoptotic cells in the GCL from E16.5 to P0 compared with controls (supplemental Fig. S2, available at www. jneurosci.org as supplemental material), suggesting that the newly generated RGCs undergo apoptosis in Barhl2-null retinas.

Previous studies have shown that BARHL2 is expressed in $\sim 33 \%$ of RGCs (Mo et al., 2004). The loss of RGCs in Barhl2 nulls is of comparable number, suggesting a cell-autonomous role of BARHL2 in RGCs. Coimmunolabeling Barhl2-null retinas using anti-POU4F2 and anti-lacZ demonstrated that 90-95\% of RGCs immunoreactive for lacZ and POU4F2 were absent in Barhl2-null retinas (Fig. $4 \mathrm{~N}-\mathrm{O}$, arrows). In contrast, the number of RGCs immunoreactive for POU4F2 alone (green) remained the same, consistent with the cell-autonomous role for BARHL2 in development and survival of a selective set of RGCs.

Although BARHL2 expression is restricted to a subset of RGCs in the adult, it may also be expressed transiently in other RGCs during development. To investigate the fate of all Barhl2 lineage RGCs, we used a lineage tracing strategy to permanently mark cells that ever expressed Barhl2. Barhl2-Cre knock-in mice were generated and expressed Cre recombinase under Barhl2 regulatory sequences. Barhl2-Cre mice were bred with the Z/EG conditional GFP reporter mice, which constitutively expresses an enhanced GFP after the Cre-mediated recombination (Novak et al., 2000), to generate Barhl2 ${ }^{\text {cre/+ }} ; Z / E G /+$ mice for the analysis of Barhl2-lineage cells in control retinas (supplemental Fig. S3, available at www.jneurosci.org as supplemental material). Similarly, Barhl2-null (Barhl2 $\left.{ }^{\text {cre/lac }} ; Z / E G /+\right)$ mice were generated from Barhl2 ${ }^{\text {cre } /+} ; Z / E G /+$ and Barhl2 ${ }^{\text {lacZ/+ }}$ heterozygotes crossing to determine Barhl2-lineage cells in the absence of Barhl2. Colabeling of GFP and POU4F2 demonstrated
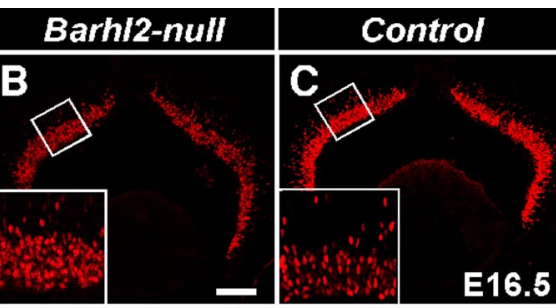

Barh/2-null
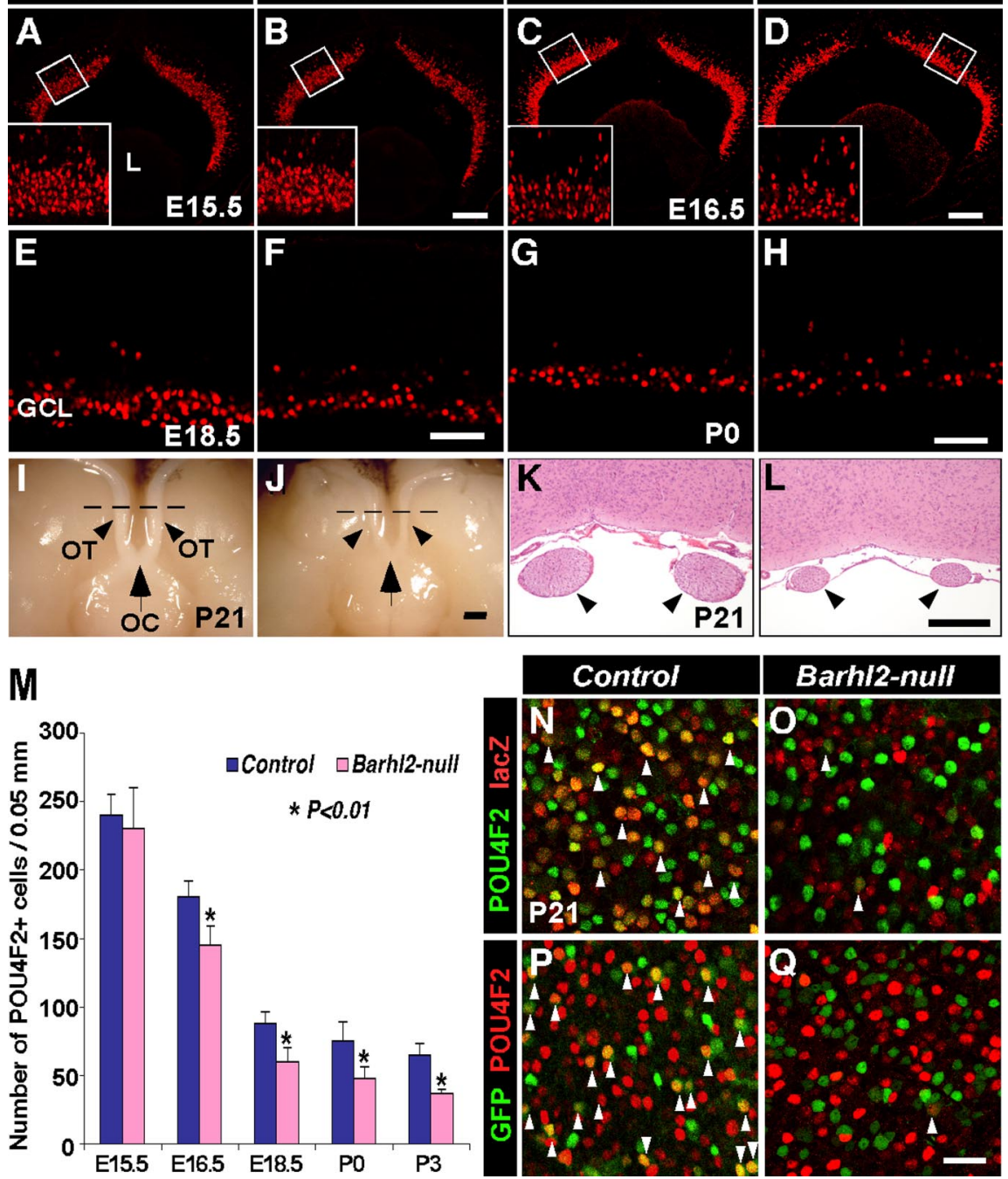

Barh/2-null
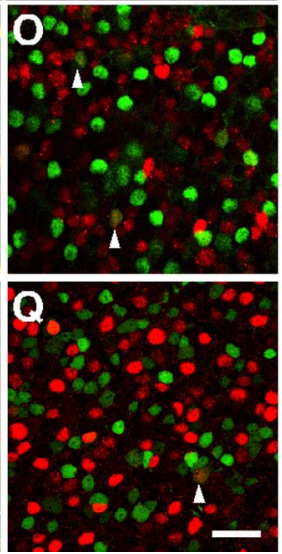

Figure 4. Loss of RGCs in Barh/2-null retina. $\boldsymbol{A}, \boldsymbol{B}$, No overt change in the number of POU4F2 immunoreactive RGCs is detected in Barh/2-null retinas at and before E15.5. $\mathbf{C} \boldsymbol{H}$, Starting at E16.5, there is a progressive reduction in RGCs in Barh/2-null retinas. Inserts show the enlarged views of boxed regions in the central retina. I, J, Ventral views of brains reveal optic nerves (arrowheads) lines in $I$ and $J$. The cross-section areas (arrowheads) of the Barh/2-null optic nerves are reduced by $\sim 33.7 \%$ compared with controls ( $n=3, p<0.001$, Student's $t$ test). M, Quantitation of RGCs immunoreactive for P0U4F2 in late embryonic stages and a severe loss of lacZ + RGCS (arrowhead; yellow) in Barh/2 null. $\boldsymbol{P}, \mathbf{Q}$, Lineage tracing analysis by immunostaining of whole-mount retinas with anti-GFP (green) and anti-POU4F2 (red) shows a severe reduction of Barh/2-lineage RGCs (arrowhead; yellow) in Barh/2 null. OC, Optic chiasm; 0T, optic tract. Scale bars: (in $\boldsymbol{B}, \boldsymbol{D}, \boldsymbol{F}$, and $\boldsymbol{H}$ ) $\boldsymbol{A}-\boldsymbol{H}, 50 \mu \mathrm{m}$; (in $\boldsymbol{L}$ ) $\boldsymbol{I}-\boldsymbol{L}, 300 \mu \mathrm{m}$; (in $\mathbf{Q}) \boldsymbol{N}-\boldsymbol{Q}, 25 \mu \mathrm{m}$.

that compared with heterozygous retina at $\mathrm{P} 21$, Barhl2-null retinas had a loss of $\sim 95 \%$ of Barhl2-lineage RGCs (Fig. 4P, Q), thus confirming the cell-autonomous role of Barhl2 and the necessity of Barhl2 in the development of RGCs.

Altered amacrine subtype composition in Barhl2-null retinas The partial loss of ACs in the INL (Fig. 2B) and the twofold increase in POU4F2 - and ISL1 + cells (presumably the displaced cholinergic ACs) in the GCL (Fig. $3 C-E$ ) prompted us to examine the subtype-specific changes of ACs in Barhl2-null retinas. Immunostaining of GAD65 for GABAergic ACs showed that the number of GABAergic ACs was significantly reduced in Barhl2null retinas by $34 \%$ compared with the controls (Fig. $5 A$ ). Immunolabeling of BHLHB5, a bHLH TF expressed in a group of GABAergic ACs (Feng et al., 2006), revealed a similar loss of 


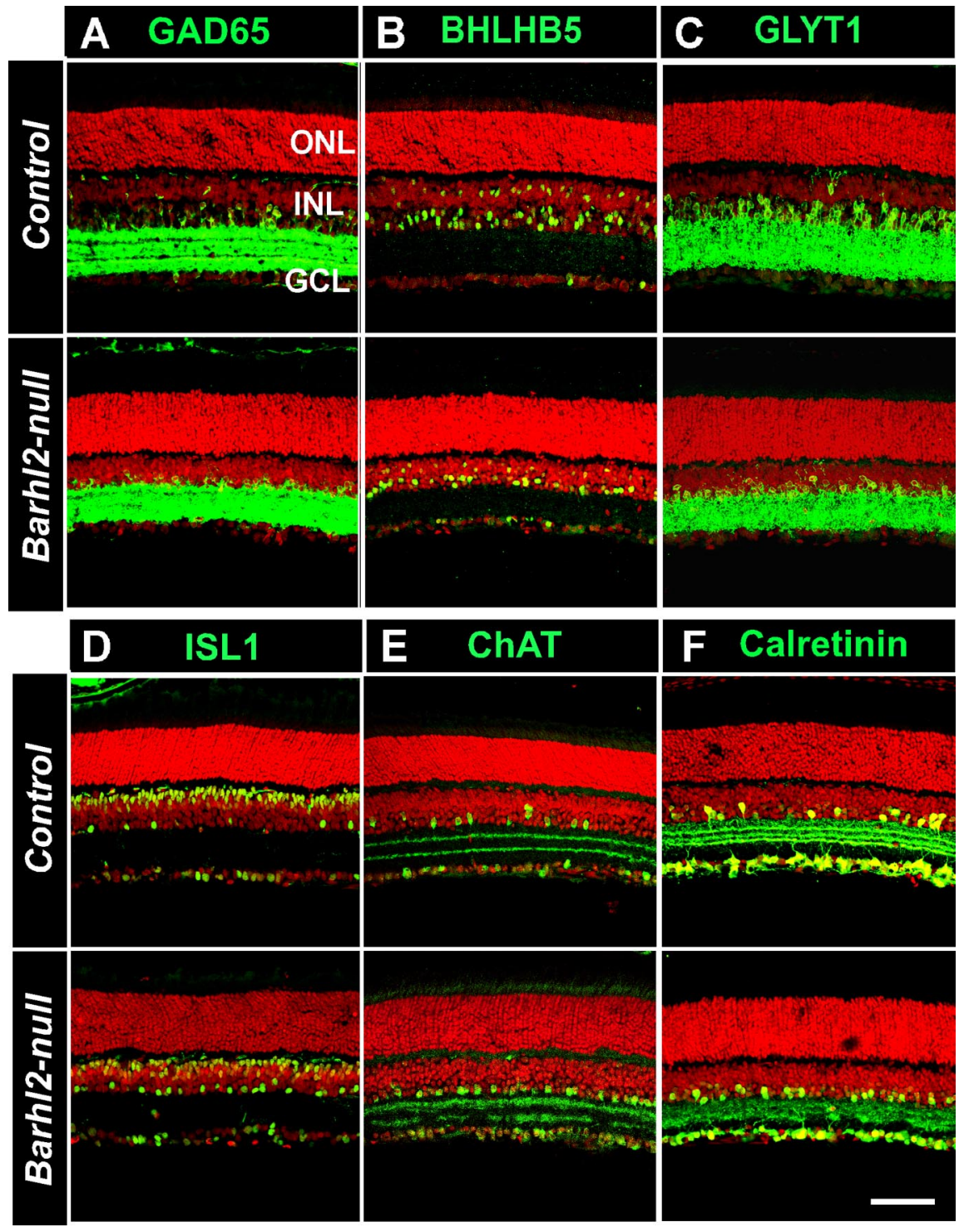

Figure 5. Subtype-specific effects on ACs Barh/2-null retina. Sections from P21 mouse retinas were immunolabeled with $A C$ subtype-specific markers (green) and nuclear counterstained with PI (red). $\boldsymbol{A}-\boldsymbol{C}$, Loss of Barh/2 leads to a severe loss of ACs immunoreactive for GAD65 ( $\boldsymbol{A}$, average number of GAD65 + ACs \pm SD: controls, $62 \pm 6, n=4 ;$ Barhl2 nulls, $39 \pm 5, n=4 ; p<$ 0.01, Student's $t$ test), Bhlhb5 (B), and GLYT1 ( $\boldsymbol{C}$, average number of GLYT1+ ACs $\pm S D$ : controls, $89 \pm 6, n=4$; Barhl2 nulls, $50 \pm 3, n=4 ; p<0.001$, Student's $t$ test). $\boldsymbol{D}-\boldsymbol{F}$, There is also an overt increase of amacrine cells immunoreactive for Isl1 ( $\boldsymbol{D}$, average number of ISL1+ ACs \pm SD: controls, $10 \pm 2, n=4 ;$ Barhl2 nulls, $18 \pm 3, n=4 ; p<0.01$, Student's $t$ test), ChAT (E), and calretinin $(\boldsymbol{F})$. Scale bar, $100 \mu \mathrm{m}$.

GABAergic ACs (Fig. 5B). Likewise, anti-GLYT1 labeling demonstrated a $40 \%$ reduction in glycinergic ACs in Barhl2-null retinas compared with the controls (Fig. 5C). In contrast, the cholinergic ACs labeled by anti-ISL1, ChAT, and calretinin in the ACL were increased (Fig. 5D-F). Whole-mount immunolabeling showed that in the ACL, ISL1 expression completely overlapped that of ChAT and that there was a threefold increase in the cholinergic amacrine cell density in Barhl2-null retinas (Fig. 6A, B). Similarly, ChAT- and ISL1-labeled displaced cholinergic ACs were increased by twofold (Fig. 6C,D).

ISL1 is expressed in developing and mature cholinergic ACs. Retina-specific deletion of Isll abolishes all cholinergic ACs (Elshatory et al., 2007). To elucidate the mechanism underlining the increase of cholinergic ACs in Barhl2-null retinas, we assessed ISL1 expression during early postnatal retinal development. We found that there were approximately twofold increases in the number of cells expressing ISL1 at the inner edge of the NBL (presumably the developing ACL) in Barhl2-null retinas at P0-P5 (Fig. 6E, F, brackets). In contrast, there was a reduction of $40 \%$ differentiating PAX6 + ACs in Barhl2-null retinas at P5 (Fig. $7 A, B$ ). Anti-BHLHB5 immunolabeling detected a similar reduction of a subset of GABAergic ACs at this stage (Fig. $7 C, D)$. Moreover, using anti-activated CASP3 immunostaining, we detected an increase of apoptotic cells in the NBL where the nascent ACs were localized (Fig. $7 E, F, J)$. Colabeling with anti-PAX6 and anti-CASP3 confirmed some of these apoptotic cells as ACs (Fig. 7G-I, arrows). Thus, loss of Barhl2 affects the amacrine subtype composition by differentially changing the genesis and survival of different amacrine subtypes.

Roles of Barhl2 in the genetic regulatory networks of ACs and RGCs

Neurod 1 and Neurod4 are redundantly required for AC genesis (Inoue et al., 2002). To define the role of Barhl2 in the genetic regulatory network of AC cell differentiation, we analyzed the expression of $\mathrm{Neu}$ rod4 and Neurod1 in control and Barhl2null retinas by in situ hybridization and observed no detectable alteration in their expression from E14.5 to P0 (Fig. 8A,B) (data not shown), suggesting that Barhl2 unlikely functions upsteam of Neurod4 and Neurod1 during AC differentiation. Rather, Barhl2 could function downstream of Neurod4 and Neurod1 in the AC differentiation pathway to negatively regulate the development of cholinergic amacrine subtype by suppressing Isl 1 .

In Drosophila, the proneural gene ato is required for the specification of the first born retinal neurons, R8 photoreceptors (Brunet and Ghysen, 1999). During retinogenesis, ato expression is sequentially restricted from the most anterior region of morphogenetic furrow to R8 founder cells. This restricted expression of ato is partially controlled by BarH1 and BarH2. Loss of BarH induces cellautonomous ectopic expression of ato (Lim and Choi, 2004). The murine orthologue of ato, Atoh7, is transiently expressed in ganglion-competent retinal precursors and is essential for RGC differentiation (Wang et al., 2001; Yang et al., 2003). Thus, we asked whether Barhl2 could negatively regulate the expression of Atoh7. As shown by in situ hybridization, loss of Barhl2 had no effect on the expression of Atoh7 (Fig. 8C). Conversely, the expression of Barhl2 was significantly downregulated in both the GCL and the inner NBL of Atoh7-null retinas at E13 (Fig. 8D). Furthermore, Barhl2 expression was greatly downregulated in Pou4f2-null retinas at E13.5 (Fig. 8E). Therefore, Barhl2 functions downstream of Atoh7-Pou 42 pathway to regulate the development of a subset of RGCs. 

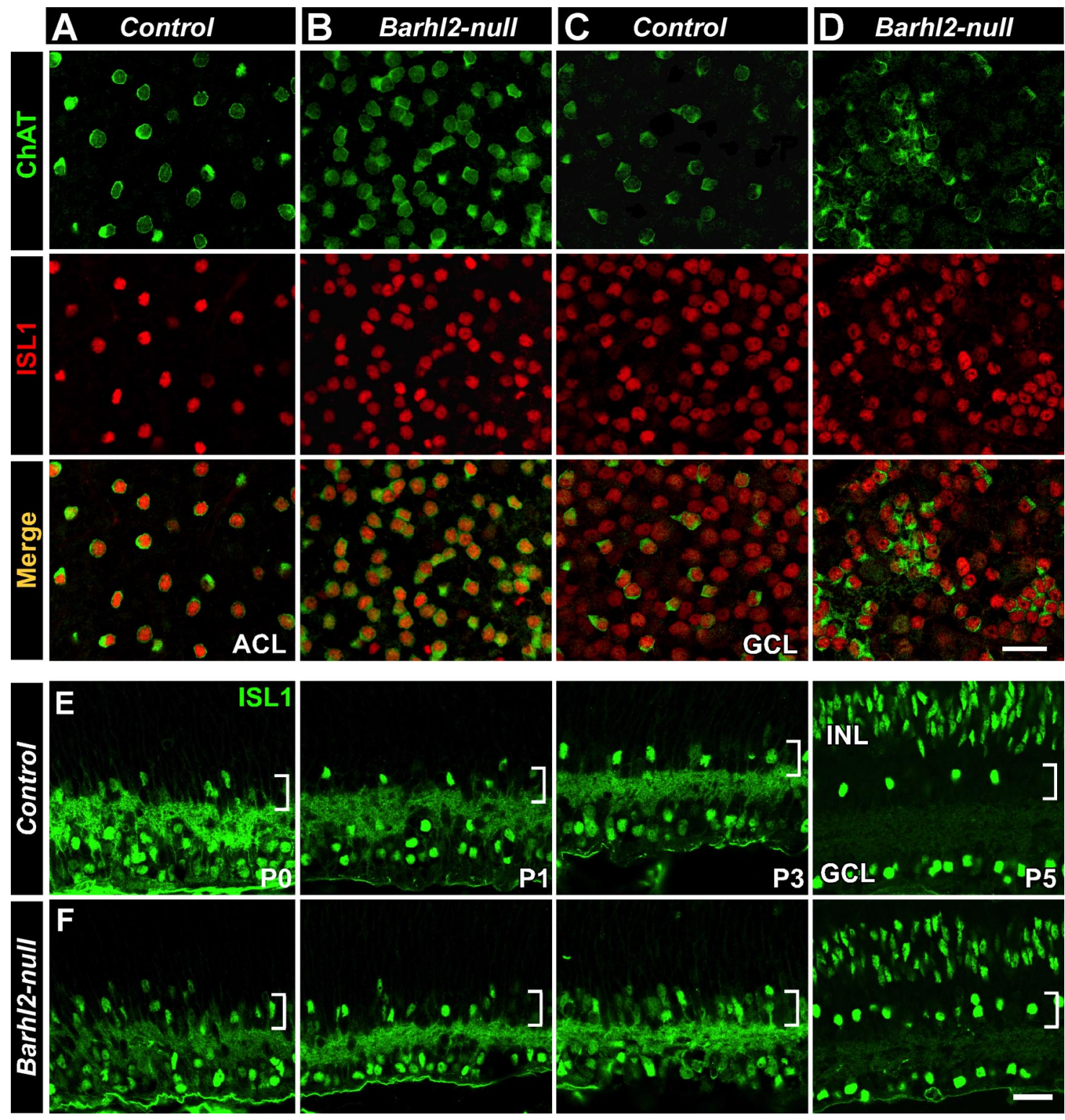

Figure 6. Cholinergic ACs are increased in Barhl2-null retinas. $A, B$, Double immunolabeling of control and Barh/2-null retinas with anti-ISL1 (green) and anti-ChAT (red) reveals a threefold increase in cholinergic ACs in the absence of Barhl2 at P21. C, D, In the GCL, there is a twofold increase in the displaced cholinergic ACs in Barhl2-null retinas. $\boldsymbol{E}, \boldsymbol{F}$, Upregulation of ISL1 expression in the ACL of Barh/2-null retina during the development of cholinergic amacrine cells (brackets). Scale bars, $25 \mu \mathrm{m}$.

Functional impairments in Barhl2-null mice

Finally, we determined the functional impacts of Barhl2 deletion on retinal cholinergic ACs by recording spontaneous and lightevoked synaptic activity of RGCs. We first examined how genetic deletion of Barhl2 affects cholinergic synaptic activity in the developing retina. We used a multielectrode array (MEA) recording to examine spontaneous RGC activity in Barhl2-null mice at P3 and $\mathrm{P} 13$ (Fig. 9A). The spontaneous retinal waves at $\mathrm{P} 3$ are mediated by nicotinic acetylcholine receptors (nAChRs) and the retinal waves at $\mathrm{P} 13$ are mediated by glutamate receptors (GluRs) (Bansal et al., 2000). The frequency of nAChR-mediated retinal waves, measured as the interwave interval, in Barhl2-null mice at P3 was $\sim 50 \%$ lower than that of age-matched controls, whereas the interwave interval of GluR-mediated retinal waves in Barhl2null mice at P13 was not different from age-matched controls (Fig. $9 B-D$ ), demonstrating that genetic deletion of Barhl2 pref-

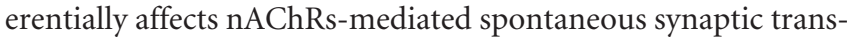
mission in developing retina.
We then examined whether genetic deletion of Barhl2 affects the light-evoked synaptic transmission specific to cholinergic ACs by examining the light-evoked responses of directional selective (DS) RGCs. DSRGCs exhibit vigorous spiking activity when a visual object moves in their preferred direction across the receptive field but minimum response when the same object sweeps across in the opposite (null) direction (compare Fig. $10 A, B$ ) because of spatially offset GABAergic inhibition from cholinergic starburst ACs (Demb, 2007). In mouse retina, most of the ON-OFF responsive RGCs are DSRGCs. We specifically examined the strength of the directional selectivity, as a measure of the specific asymmetric inhibitory synaptic transmission between cholinergic starburst ACs and DSRGCs, and the peak frequency of light-evoked responses, as a measure of the strength of overall inhibitory synaptic inputs, of ON-OFF DSRGCs in Barhl2-null mice. Consistent with an increase of GABAergic synaptic inputs from cholinergic starburst ACs, the average peak frequency of light-evoked responses of ON-OFF DSRGCs in 

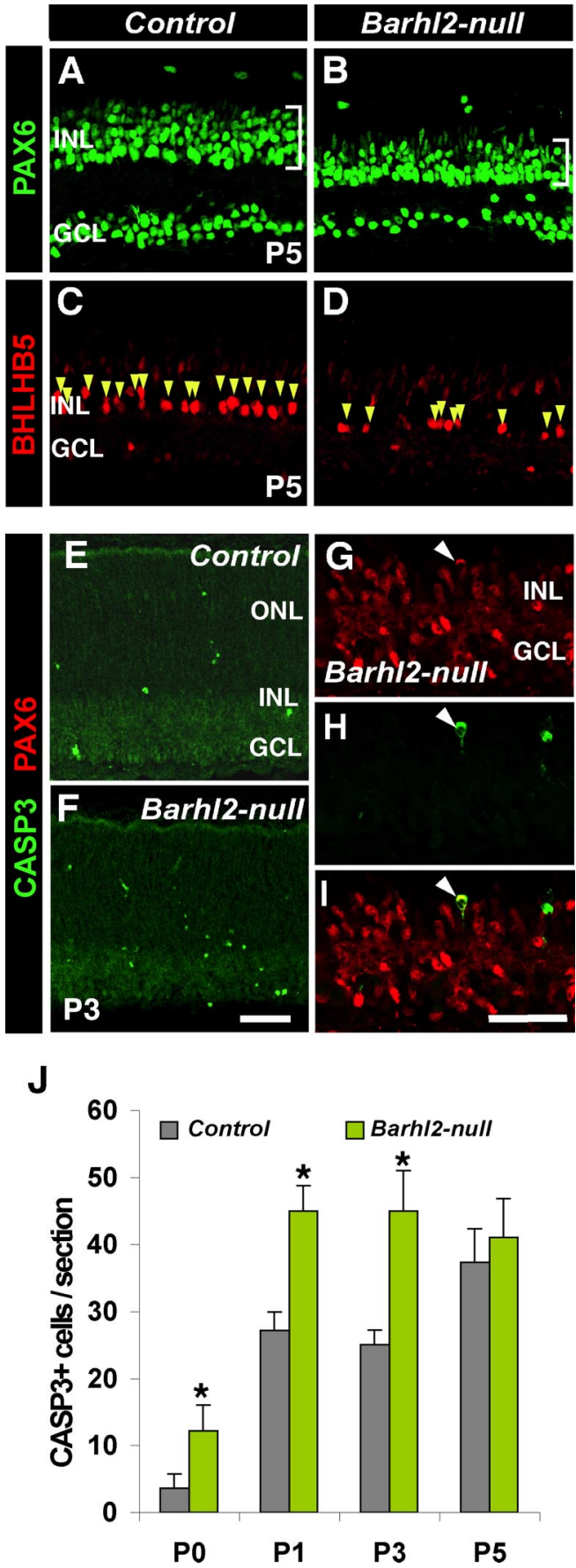

Figure 7. Barh/2-null retinas exhibit an elevated cell apoptosis in ACS. $A, B$, Immunostaining of retina sections with PAX6 reveals a significant loss of PAX6 + amacrine cells (brackets) in Barh/2-null retina at P5. C, D, Anti-BHLHB5 immunostaining shows a decrease in a selective group of GABAergic amacrine cells in Barh/2-null retina (yellow arrowhead). $\boldsymbol{E}, \boldsymbol{F}$, Anti-activated CASP3 immunostaining shows an increase of apoptotic cells in the INL of Barh/2-null retina at P3. G-I, Expression of PAX6 (red) in apoptotic cells shown by anti-CASP3 (green) in Barh/2-null retina (yellow arrowhead) at P3.J, Quantification of apoptotic cells in the INL of the developing retina by averaging activated caspase3-positive cells in 10 sections per retina. The differences in apoptoticcell numbers in control and Barhl2-null retina are significant from P0 to P5. ${ }^{*}$ test $p<$ 0.01. Scale bars, $100 \mu \mathrm{m}$.

Barhl2-null mice is significantly lower than that of controls (Fig. $10 D, E)$. In contrast, the average peak frequency of light-evoked responses of ON RGCs in Barhl2-null mice, which are dominated by non-DSRGCs, is not different from that of controls (Fig. 10C). Interestingly, the peak frequency of light-evoked responses of the null direction of ON-OFF DSRGCs is more significantly suppressed in Barhl2-null mice, which resulted in a slight increase of the strength of directional selectivity (Fig. 10F). This is consistent with an asymmetric GABAergic inhibition from cholinergic starburst ACs to ON-OFF DSRGCs with more effective inhibition on the null direction. Together, these results strongly support the notion that genetic deletion of Barhl2 specifically impaired GABAergic synaptic transmission mediated by cholinergic starburst ACs in mouse retina.

\section{Discussion}

In this report, we have generated Barhl2-null mice and demonstrated Barhl2's essential role in the development of multiple retinal cell types. Loss of Barhl2 results in a marked decrease of RGCs. During the development of ACs, Barhl2-null mutation affects the development of distinct AC subtypes. Loss of Barhl2 leads to the partial loss of glycinergic and GABAergic ACs, a significant increase in cholinergic ACs, and altered synaptic inputs of cholinergic ACs to RGCs in both immature and mature retinas. Gene expression studies show that Barhl2-null mutation leads to the upregulation of ISL1, the key regulator of cholinergic ACs in the INL and the GCL. Furthermore, we have demonstrated that Barhl2 functions downstream of Atoh7-Pou $4 f 2$ pathway in RGC development and likely downstream of Neurod1 and Neurod4 in differentiating ACs. The differential effect of Barhl2null mutation on each retinal neuronal subtype implies a mechanism that BARHL2 and other TFs form a unique combinatorial code in each subtype and regulate the acquisition of subtype identities.

\section{Essential roles of Barhl2 in the development of RGCs}

Previous studies have demonstrated that ATOH7 and POU4F2 constitute the essential genetic cascade of RGC development. ATOH7 determines the RGC competence of retinal precursors (Brown et al., 2001; Wang et al., 2001; Yang et al., 2003), whereas POU4F2 is required for the terminal differentiation of RGCs including axon growth and pathfinding as well as survival (Gan et al., 1996, 1999). The downregulation of Barhl2 expression in Atoh7-null and Pou4f2-null retinas (Fig. 8D,E) and the partial loss of RGCs in Barhl2-null retinas suggest that Barhl2 functions downstream of Atoh7 and Pou4f2 to control the differentiation of a subset of RGCs. Similarly, in Xenopus, Xbh1 (orthologous to BARHL2) is reported to interact with Xath5 and Xath3 and act as a late transcriptional repressor downstream of Xath 5 in the RGCs development (Poggi et al., 2004). Although Barhl2 is a direct downstream target of MATH1 in the spinal cord (Saba et al., 2005), it remains to be determined whether ATOH7 directly regulates Barhl2 in retinas. However, the late onset of Barhl2 expression at E13.5 and the downregulation of Barhl2 in Pou4f2-null retinas suggest that Barhl2 is an indirect downstream target of Atoh7 during RGC development.

In Barhl2-null mice, there is no change in the initial formation and migration of the nascent RGCs (POU4F2+) to the GCL, but there is a progressive reduction of $\sim 35 \%$ RGCs accompanied by the reduced thickness of optic nerves (Figs. 2-4), suggesting that Barhl2 is dispensable for RGC genesis but necessary for RGC maturation and survival. Targeted deletion of Barhl2 could result in the RGC defects through two possible mechanisms. First, BARHL2 could function cell autonomously to control the maturation and survival of the BARHL2-expressing RGCs. Without BARHL2, these RGCs fail to develop properly and undergo apoptosis. Such cell-autonomous effects were previously observed during retinal development in mice null for several TFs. For ex- 

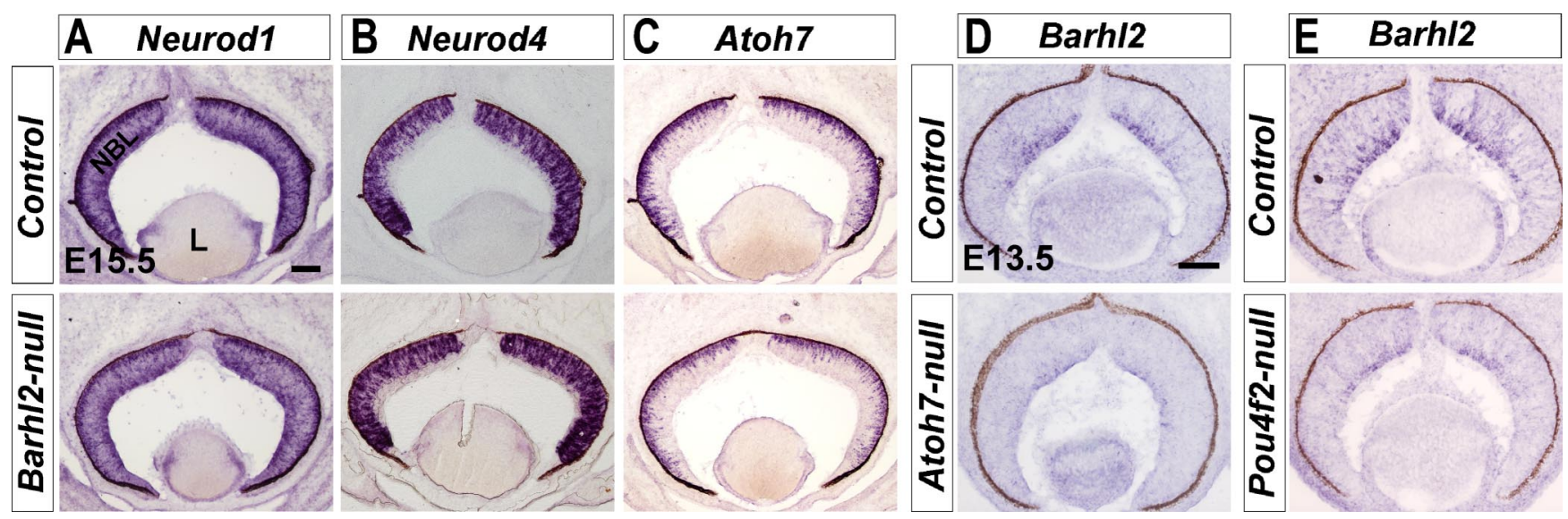

Figure 8. Analysis of potential upstream and downstream genes of Barh/2. A-C, The expression profiles of retinogenic bHLH genes, Atoh7, Neurod4, and Neurod1, are unaffected in Barh/2-null retinas by in situ hybridization at E16.5. D, E, Barh/2 expression is downregulated in Atoh7-null and Pou4f2-null retinas. In situ hybridization reveals a reduced expression of Barh/2 in Atoh7-null (D) and Pou4f2-null $(\boldsymbol{E})$ retinas at E13.5. L, Lens; NBL, neuroblast layer retina. Scale bars, $100 \mu \mathrm{m}$.

ample, BHLHB4 is specifically expressed in rod-bipolar cells, and Bhlhb4 knockout abolishes the rod-bipolar cell population (Bramblett et al., 2004). Similarly, ISL1 is required cell autonomously for the development of bipolar cells and cholinergic ACs, and POU4F2 and SHH act cell autonomously in RGCs (Elshatory and Gan, 2008; Pan et al., 2008; Sanchez-Camacho and Bovolenta, 2008). Alternatively, BARHL2 could function in a non-cellautonomous manner. Loss of Barhl2 may not affect the RGCs of BARHL2 + subset. Rather, it indirectly influences the maturation and survival of the RGCs that never express BARHL2. Non-cellautonomous effect was previously seen in retinas transfected with SMO-M2, a constitutive active allele of Smoothened, that SMO-M2 expression inhibits rhodopsin expression in uninfected photoreceptor cells (Yu et al., 2006). Interestingly, the extent of RGC loss is in proportion to the number of RGCs expressing Barhl2 as shown by the colocalization of POU4F2 and lacZ in Barhl2 ${ }^{\text {lacZ/+ }}$, suggesting a cell-autonomous role of BARHL2 in the maturation and survival of some RGCs. We performed a lineage analysis using Barhl2-Cre knock-in allele (Fig. $4 \mathrm{~N}-\mathrm{Q}$ ) and demonstrated the loss of RGCs that are mostly from Barhl2-cell lineage, thus confirming the cell-autonomous role of BARHL2 in RGCs.

The roles of Barhl2 in the subtype specification of ACs

ACs form synapses with bipolar cells and RGCs and modulate the signal processing by excitatory and inhibitory effects. The development of ACs depends on several TFs. FOXN4 regulates amacrine genesis by activating the expression of amacrine differentiation factors, Neurod4 and Neurod1 (Li et al., 2004). PTF1, a bHLH TF, is essential for the specification of amacrine and horizontal cells, acting as a primary target of FOXN4. These above TFs do not regulate the subtype specification of ACs, and little is known about how the mammalian retina generates the numerous different subtypes of ACs. PAX6, a key regulator in the early retinal development, is expressed in differentiating ACs and positively regulates the formation of glycinergic ACs (Marquardt et al., 2001). Recently, we show that BHLHB5 and ISL1 are uniquely expressed and required for the differentiation of GABAergic (Feng et al., 2006) and cholinergic (Elshatory et al., 2007) amacrine subtypes, respectively. Although Barhl2 is expressed in all amacrine subtypes, loss of Barhl2 does not affect the initial formation of ACs expressing Neurod1 and Neurod4. Rather, it has subtype-specific effects on ACs, resulting in a $40 \%$ loss of glycin- ergic and GABAergic ACs and in a significant increase of cholinergic ACs. These differential effects imply that BARHL2's role in amacrine cell differentiation depends on the unique cellular environment of each amacrine subtypes. Therefore, our data indicate that the subtype specification of ACs is determined after the acquisition of pan-amacrine properties and is governed by the unique combinatorial activities of overlapping transcriptional activators and repressors.

The combinatorial codes of TFs play many roles in embryonic development. It is first revealed in Drosophila that the overlapping expression of transcriptional activators and repressors in early embryos generates the combinatorial TF codes essential for the formation of segmentation stripes (Stanojevic et al., 1991). During vertebrate neurogenesis, the combinatorial actions of $\mathrm{HD}, \mathrm{bHLH}$, and LIM-HD proteins regulate the specification of neural cell types in the spinal cord (Jessell, 2000; Bertrand et al., 2002). In ventral spinal cord, for example, the partial overlapping expression of PAX6, NKX6.1, and NKX2.2, and their mutual repressive activities, define a progenitor domain from which motor neurons are generated (Tanabe et al., 1998). The function of the combinatorial activities of partially overlapping transcriptional activators and repressors is similarly proposed to regulate the neurogenesis during cortical development (O'Leary and $\mathrm{Na}$ kagawa, 2002). Recently, we show that Bhlhb5 is expressed in neurons in layers II-V of somatosensory and visual cortex and that targeted deletion of Bhlhb5 leads to altered gene expression patterns in area- and layer-specific manners. For example, the expression of ROR $\beta$ in layer IV of Bhlhb5 nulls is downregulated in somatosensory but not visual cortex where Bhlhb5 is also highly expressed and is required for the expression of Eph7A. Additionally, BHLHB5 regulates the expression of Ephrin-A5 in layers IV-V but not layers II-III of somatosensory cortex despite BHLHB5's expression in layers II-V. These results provide direct evidence for this combinatorial code model within the developing cortex (Joshi et al., 2008).

Ectopic expression of bHLH and HD TFs in different combinations in retina are capable to uniquely enhance or suppress the differentiation of the six major retinal cell types (Wang and Harris, 2005). Here, we show that although Barhl2 is expressed in horizontal, amacrine, and ganglion cells, targeted disruption of Barhl2 does not appear to affect horizontal cells but leads to the altered subtype composition of ACs and a partial loss of RGCs. Despite Barhl2's expression in the three AC subtypes, loss of 

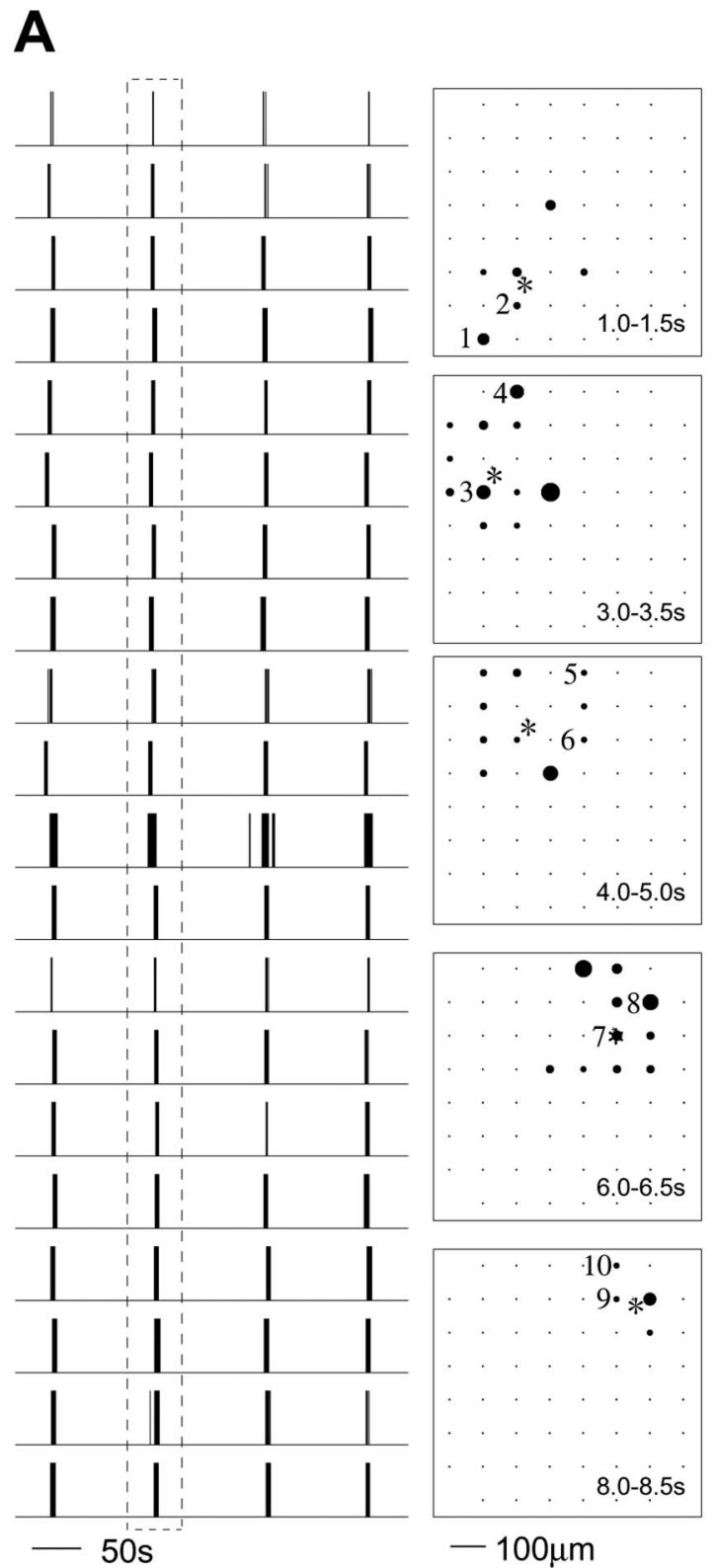
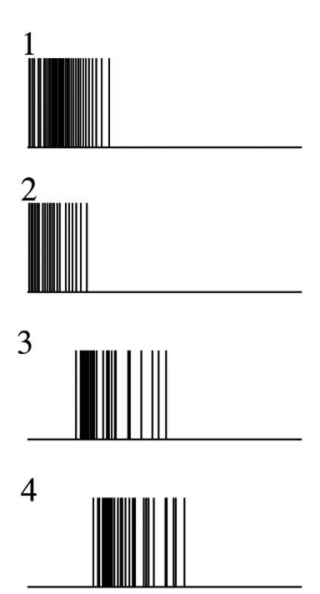

\section{5}

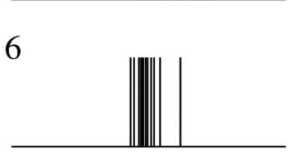

7

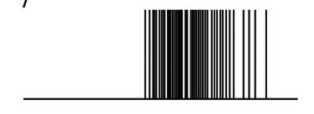

8
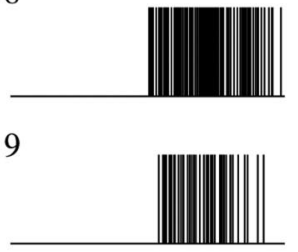

10

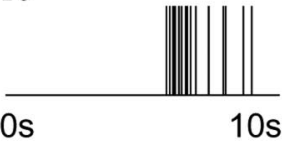

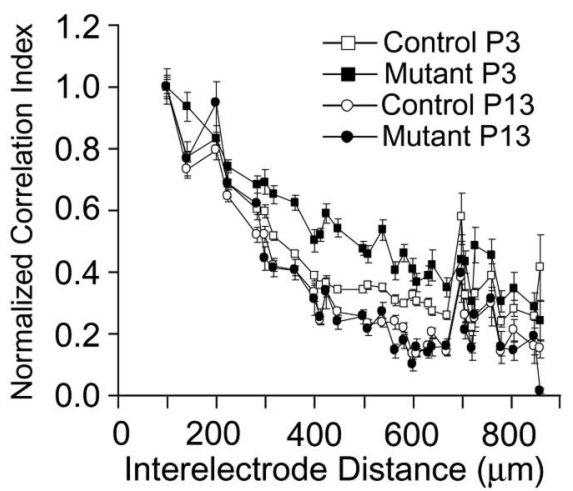
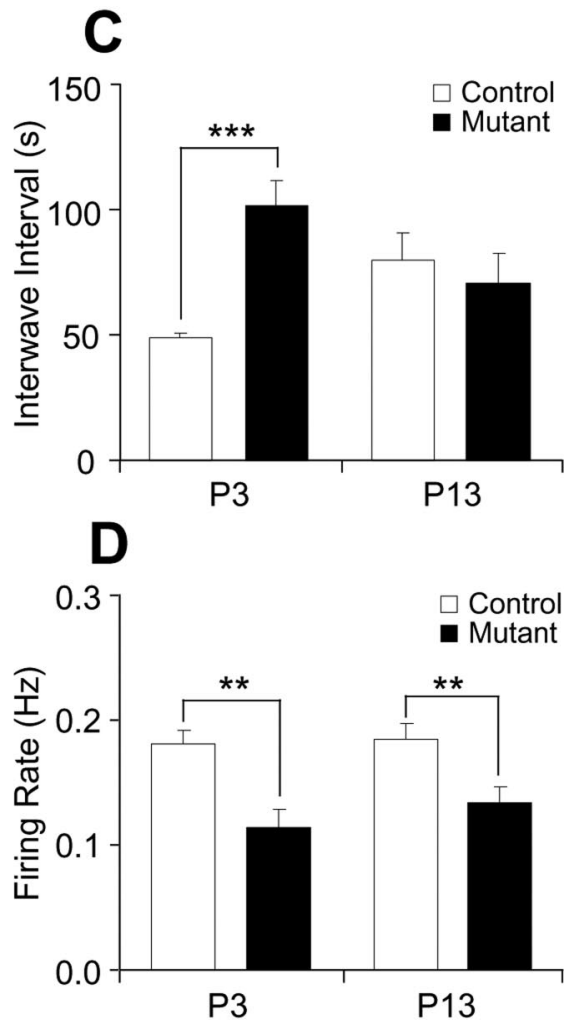

Figure 9. The retinal waves mediated by cholinergic synaptic transmission were affected in Barh/2-null mice. Spontaneous retinal waves were recorded from P3 and P13 Barh/2-null mice and age-matched WT controls. A, Example of retinal waves recorded from a P3 WT retina. Left column, Spike trains of 20 neurons. Center column, A diagram of the array showing a propagating retinal wave. Each frame shows the mean firing rate of each cell (averaged $>0.5 \mathrm{~s}$ ). The small black dots represent the position of electrodes. Each black circle represents one cell, with the radius of the circle proportional to its firing rate. Right column, Spike trains of 10 neurons selected from the time window shown in the left column and the position of each neuron is represented with the number in the center column. $\boldsymbol{B}$, Normalized correlation index as a function of distance between the recording electrodes from both WT and Barh/2-null mice at the ages of P3 and P13, respectively. $\boldsymbol{C}$, Average interwave interval of the spontaneous retinal waves of both Barh/2-null mice and age-matched WT controls, showing that waves are much less frequent in Barh/2-null mice in the first postnatal week. $D$, Average firing rate of RGCs of Barh/2-null mice and age-matched WT controls at the ages of P3 and P13, respectively.

Barhl2 results in a significant reduction of glycinergic and GABAergic ACs accompanied by a substantial increase in cholinergic ACs (Fig. 5-7). Our results indicate that BARHL2 and other yet to be identified TFs form unique combinatorial codes in each AC subtype, a mechanism comparable with that of BHLHB5 in cortex, and thus provide an example of combinatorial TF codes that specify AC subtypes. Future identification of additional regulators of AC subtype differentiation will increasingly elucidate the precise mechanism by which combinatorial TF codes determine the AC subtypes. In RGCs, the expression of BARHL2 in combination with other RGC-specific factors, such as POU4F2 and ISL1, defines a particular group of RGCs. Future morphological, physiological, and functional analyses of the Barhl2-lineage
RGCs will shed light on their subtype identity and difference from other RGCs expressing POU4F2 and ISL1 but not BARHL2.

Published studies have shown that forced expression of Barhl2 by viral infection in mouse retinas promotes the differentiation of glycinergic ACs but has no effect on the differentiation of GABAergic ACs, RGCs, and horizontal cells (Mo et al., 2004). The reported nonessential role of BARHL2 in horizontal cells is consistent with our targeted disruption study and with our model that retinal cell types are regulated by unique combinatorial TF codes. The increase in glycinergic ACs in Barhl2 over-expression retina also agrees with the reduction in glycinergic ACs in Barhl2null retinas, demonstrating the essential role of Barhl2 in these ACs. However, the effect of ectopic Barhl2 expression on 
GABAergic ACs and RGCs appears to differ from the loss-of-function data presented here showing that Barhl2-null mutation results in a decrease in RGCs, GABAergic ACs, and an increase in cholinergic ACs. One likely explanation is that the viral infection is performed in neonatal retinas at $\mathrm{P} 0$ after the differentiation of most ACs and RGCs and likely after BARHL2 executes its roles. The reduction of RGCs in Barhl2-null retinas occurs mostly during late embryogenesis (Fig. 4), when BARHL2 is critically required and functions in a proper combination with other TFs in RGCs. In the BARHL2 gainof-function experiments, BARHL2 is expressed in postnatal retinas. Possibly, attributable to the lack of necessary combinatorial TF codes, the ectopic, postnatal expression of BARHL2 has no detectable effect on RGCs and the GABAergic ACs. Further experiments with the BARHL2 gain of function in embryonic retinas will help to address these seemingly different observations in GABAergic ACs.

\section{References}

Auerbach W, Dunmore JH, Fairchild-Huntress V, Fang Q, Auerbach AB, Huszar D, Joyner AL (2000) Establishment and chimera analysis of $129 / \mathrm{SvEv}$ - and C57BL/6-derived mouse embryonic stem cell lines. Biotechniques 29: 1024-1028, 1030, 1032.

Bansal A, Singer JH, Hwang BJ, Xu W, Beaudet A, Feller MB (2000) Mice lacking specific nicotinic acetylcholine receptor subunits exhibit dramatically altered spontaneous activity patterns and reveal a limited role for retinal waves in forming $\mathrm{ON}$ and $\mathrm{OFF}$ circuits in the inner retina. J Neurosci 20:7672-7681.

Bertrand N, Castro DS, Guillemot F (2002) Proneural genes and the specification of neural cell types. Nat Rev Neurosci 3:517-530.

Bramblett DE, Pennesi ME, Wu SM, Tsai MJ (2004) The transcription factor Bhlhb4 is required for rod bipolar cell maturation. Neuron 43:779-793.

Brown NL, Patel S, Brzezinski J, Glaser T (2001) Math5 is required for retinal ganglion cell and optic nerve formation. Development 128:2497-2508

Brunet JF, Ghysen A (1999) Deconstructing cell determination: proneural genes and neuronal identity. Bioessays 21:313-318.

Demb JB (2007) Cellular mechanisms for direction selectivity in the retina. Neuron 55:179-186.

Elshatory Y, Gan L (2008) The LIM-homeobox gene Islet-1 is required for the development of restricted forebrain cholinergic neurons. J Neurosci 28:3291-3297.

Elshatory Y, Everhart D, Deng M, Xie X, Barlow RB, Gan L (2007) Islet-1 controls the differentiation of retinal bipolar and cholinergic amacrine cells. J Neurosci 27:12707-12720.

Feng L, Xie X, Joshi PS, Yang Z, Shibasaki K, Chow RL, Gan L (2006) Requirement for Bhlhb5 in the specification of amacrine and cone bipolar subtypes in mouse retina. Development 133:4815-4825.

Gan L, Xiang M, Zhou L, Wagner DS, Klein WH, Nathans J (1996) POU domain factor Brn-3b is required for the development of a large set of retinal ganglion cells. Proc Natl Acad Sci U S A 93:3920-3925.

Gan L, Wang SW, Huang Z, Klein WH (1999) POU domain factor Brn-3b is

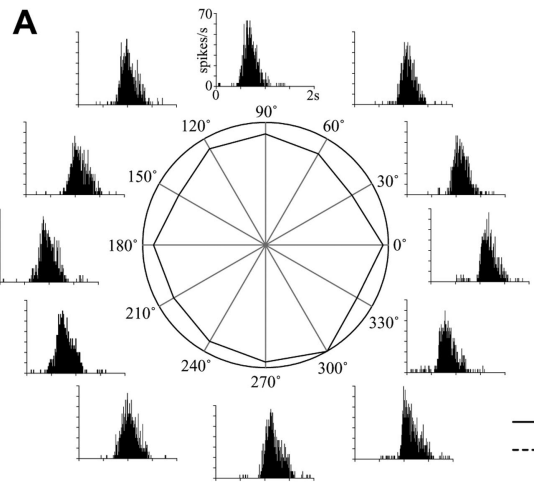

ON RGC: ON DSI $=0.022$
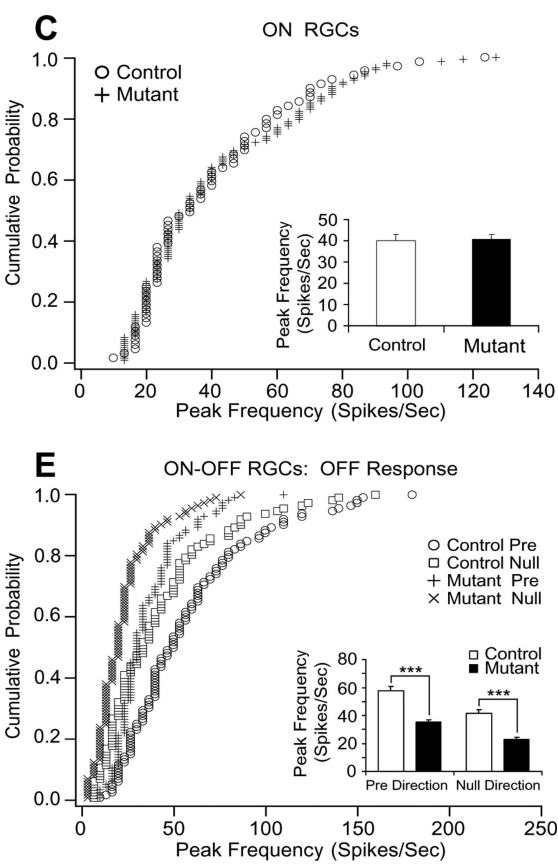

Figure 10. Light-evoked responses of ON-OFF DSRGCs are impaired in Barh/2-null mice. Responses to the moving bars of 12 different directions were recorded from the cells located in the RGC layer of P15 Barh/2-null mice and age-matched WT controls. The cells were identified as $\mathrm{ON}, 0 \mathrm{FF}$, and $\mathrm{ON}-\mathrm{OFF}$ cells based on the patterns of light responses, and the DSI and peak frequency of (center) and peristimulus response histograms to the moving bars at 12 different directions of a non-DS ON cell from a P15 WT frequencies. $E$, Cumulative distribution curves of peak frequency of the preferred and null directions of $0 \mathrm{FF}$ responses of $0 \mathrm{~N}-0 \mathrm{FF}$ DSRGCS of Barh/2-null mice and WT controls. Inset, Average peak frequencies. F, Average DSI of ON and OFF responses of ON-OFF DSRGCS of Barh/2-null mice and WT controls. essential for retinal ganglion cell differentiation and survival but not for initial cell fate specification. Dev Biol 210:469-480.

Higashijima S, Kojima T, Michiue T, Ishimaru S, Emori Y, Saigo K (1992) Dual Bar homeo box genes of Drosophila required in two photoreceptor cells, R1 and R6, and primary pigment cells for normal eye development. Genes Dev 6:50-60.

Inoue T, Hojo M, Bessho Y, Tano Y, Lee JE, Kageyama R (2002) Math3 and NeuroD regulate amacrine cell fate specification in the retina. Development 129:831-842.

Jessell TM (2000) Neuronal specification in the spinal cord: inductive signals and transcriptional codes. Nat Rev Genet 1:20-29.

Joshi PS, Molyneaux BJ, Feng L, Xie X, Macklis JD, Gan L (2008) Bhlhb5 regulates the postmitotic acquisition of area identities in layers II-V of the developing neocortex. Neuron 60:258-272. 
Kong JH, Fish DR, Rockhill RL, Masland RH (2005) Diversity of ganglion cells in the mouse retina: unsupervised morphological classification and its limits. J Comp Neurol 489:293-310.

Kuida K, Zheng TS, Na S, Kuan C, Yang D, Karasuyama H, Rakic P, Flavell RA (1996) Decreased apoptosis in the brain and premature lethality in CPP32-deficient mice. Nature 384:368-372.

Li S, Mo Z, Yang X, Price SM, Shen MM, Xiang M (2004) Foxn4 controls the genesis of amacrine and horizontal cells by retinal progenitors. Neuron 43:795-807.

Lim J, Choi KW (2004) Induction and autoregulation of the anti-proneural gene Bar during retinal neurogenesis in Drosophila. Development 131:5573-5580.

Lin B, Wang SW, Masland RH (2004) Retinal ganglion cell type, size, and spacing can be specified independent of homotypic dendritic contacts. Neuron 43:475-485.

Marquardt T, Ashery-Padan R, Andrejewski N, Scardigli R, Guillemot F, Gruss P (2001) Pax6 is required for the multipotent state of retinal progenitor cells. Cell 105:43-55.

Masland RH (2001a) The fundamental plan of the retina. Nat Neurosci 4:877-886.

Masland RH (2001b) Neuronal diversity in the retina. Curr Opin Neurobiol 11:431-436.

Mo Z, Li S, Yang X, Xiang M (2004) Role of the Barhl2 homeobox gene in the specification of glycinergic amacrine cells. Development 131:1607-1618.

Novak A, Guo C, Yang W, Nagy A, Lobe CG (2000) Z/EG, a double reporter mouse line that expresses enhanced green fluorescent protein upon Cremediated excision. Genesis 28:147-155.

O'Leary DD, Nakagawa Y (2002) Patterning centers, regulatory genes and extrinsic mechanisms controlling arealization of the neocortex. Curr Opin Neurobiol 12:14-25.

Pan L, Yang Z, Feng L, Gan L (2005) Functional equivalence of Brn3 POUdomain transcription factors in mouse retinal neurogenesis. Development 132:703-712.

Pan L, Deng M, Xie X, Gan L (2008) ISL1 and BRN3B co-regulate the differentiation of murine retinal ganglion cells. Development 135:1981-1990.

Patterson KD, Cleaver O, Gerber WV, White FG, Krieg PA (2000) Distinct expression patterns for two Xenopus Bar homeobox genes. Dev Genes Evol 210:140-144.
Poggi L, Vottari T, Barsacchi G, Wittbrodt J, Vignali R (2004) The homeobox gene Xbh1 cooperates with proneural genes to specify ganglion cell fate within the Xenopus neural retina. Development 131:2305-2315.

Saba R, Johnson JE, Saito T (2005) Commissural neuron identity is specified by a homeodomain protein, Mbh1, that is directly downstream of Math1. Development 132:2147-2155.

Sanchez-Camacho C, Bovolenta P (2008) Autonomous and nonautonomous Shh signalling mediate the in vivo growth and guidance of mouse retinal ganglion cell axons. Development 135:3531-3541.

Stanojevic D, Small S, Levine M (1991) Regulation of a segmentation stripe by overlapping activators and repressors in the Drosophila embryo. Science 254:1385-1387.

Tanabe Y, William C, Jessell TM (1998) Specification of motor neuron identity by the MNR2 homeodomain protein. Cell 95:67-80.

Tian N, Copenhagen DR (2003) Visual stimulation is required for refinement of ON and OFF pathways in postnatal retina. Neuron 39:85-96.

Torborg CL, Hansen KA, Feller MB (2005) High frequency, synchronized bursting drives eye-specific segregation of retinogeniculate projections. Nat Neurosci 8:72-78.

Wang JC, Harris WA (2005) The role of combinational coding by homeodomain and bHLH transcription factors in retinal cell fate specification. Dev Biol 285:101-115.

Wang SW, Kim BS, Ding K, Wang H, Sun D, Johnson RL, Klein WH, Gan L (2001) Requirement for math5 in the development of retinal ganglion cells. Genes Dev 15:24-29.

Wong RO, Meister M, Shatz CJ (1993) Transient period of correlated bursting activity during development of the mammalian retina. Neuron 11:923-938.

Xiang M, Zhou L, Peng YW, Eddy RL, Shows TB, Nathans J (1993) Brn-3b: a POU domain gene expressed in a subset of retinal ganglion cells. Neuron 11:689-701.

Yang Z, Ding K, Pan L, Deng M, Gan L (2003) Math5 determines the competence state of retinal ganglion cell progenitors. Dev Biol 264:240-254.

Young RW (1985) Cell differentiation in the retina of the mouse. Anat Rec 212:199-205.

Yu C, Mazerolle CJ, Thurig S, Wang Y, Pacal M, Bremner R, Wallace VA (2006) Direct and indirect effects of hedgehog pathway activation in the mammalian retina. Mol Cell Neurosci 32:274-282. 